Attention to the diet factor is another complementary method of management of chronic headache, especially for migraine headaches. Long-term prophylactic drug therapy should be avoided until headache-precipitating trigger factors, including dietary factors have been excluded (Millichap JG, Yee MM. The diet factor in pediatric and adolescent migraine. Pediatr Neurol 2003;28:9-15).

Premonitory symptoms in migraine. Electronic diaries were used in a 3-month multicenter study to record nonheadache symptoms before, during, and after migraine, that might predict an attack. Patients correctly predicted migraine headaches from $72 \%$ of diary entries with premonitory symptoms. Nonheadache, premonitory symptoms included feeling tired $(72 \%)$, difficulty concentrating (51\%), and stiff neck $(50 \%)$. Migraineurs who report premonitory symptoms that impair daily activities can accurately predict an impending attack. (Giffin NJ, Ruggiero L, Lipton RB, et al. Neurology March 25, 2003;60:935-940).

\title{
SPORADIC HEMIPLEGIC MIGRAINE: A SEPARATE ENTITY
}

The clinical characteristics of 105 patients with sporadic hemiplegic migraine (SHM) were compared with those of patients with migraine with typical aura (MA) and patients with familial hemiplegic migraine (FHM) in a study at the Danish Headache Center, Glostrup Hospital, Gentofte Hospital, University of Copenhagen, and the John F Kennedy Institute, Denmark. In patients with SHM, 72\% had 4 typical aura symptoms: visual, sensory, aphasic, and motor. Compared with MA, the duration of each aura symptom was prolonged and bilateral symptoms were more frequent. Symptoms typical of basilar migraine occurred during attacks of SHM in $72 \%$ of cases. SHM has symptoms identical to FHM and different from MA. The aura is usually followed by the headache in SHM, similar to FHM but not MA. Sporadic hemiplegic migraine should be classified with FHM and is separate from migraine with aura. The age of onset is under 15 years in $40 \%$ of cases, and one third have an onset between 10 and 14 years. Females outnumber males, 4 to 1 . Only $3 \%$ have attacks after age 52 . (Thomsen LL, Ostergaard E, Olesen J, Russell MB. Evidence for a separate type of migraine with aura. Sporadic hemiplegic migraine. Neurology Feb (2 of 2) 2003;60:595-601). (Reprints: Dr Lise L Thomsen, The Danish Headache Center, University of Copenhagen, Department of Neurology, Glostrup Hospital, Ndr Ringvej 57, DK-2600 Glostrup, Denmark).

COMMENT. In an editorial, Goadsby PJ of Queen Square, London, comments that the extent of investigation in a case of sporadic hemiplegic migraine should be exponentially increased as the length of the aura exceeds 60 minutes, but it is reassuring to know that the aura may be prolonged and can be benign. (Neurology 2003;60:536-537). About two thirds have symptoms lasting no longer than an hour, but in one third the aura is prolonged, $8 \%$ longer than a day. Clinical characteristics include sensory symptoms in $98 \%$, and brainstem symptoms in $75 \%$. Diagnostic criteria require motor symptoms and at least one visual, sensory, or speech symptom, resembling basilar migraine. Differential diagnoses of SHM include epilepsy (Todd paresis), stroke, systemic lupus, metabolic disorders, and inherited disorders such as mitochondrial myopathy. The workup should include the elimination of these and other disorders with similar symptoms. 